\title{
Distance-Dependent Scaling of AMPARs Is Cell-Autonomous and GluA2 Dependent
}

\author{
Seth L. Shipman, ${ }^{1,2}$ Bruce E. Herring, ${ }^{1}$ Young Ho Suh, ${ }^{3}$ Katherine W. Roche, ${ }^{3}$ and Roger A. Nicoll ${ }^{1}$ \\ ${ }^{1}$ Departments of Cellular and Molecular Pharmacology and Physiology and ${ }^{2}$ Neuroscience Graduate Program, University of California San Francisco, San \\ Francisco, California 94158, and ${ }^{3}$ National Institute of Neurological Disorders and Stroke, U.S. National Institutes of Health, Bethesda, Maryland 20892
}

The extensive dendritic arbor of a pyramidal cell introduces considerable complexity to the integration of synaptic potentials. Propagation of dendritic potentials is largely passive, in contrast to regenerative axonal potentials that are maintained by voltage-gated sodium channels, leading to a declination in amplitude as dendritic potentials travel toward the soma in a manner that disproportionally affects distal synaptic inputs. To counteract this amplitude filtering, Schaffer collateral synapses onto CA1 pyramidal cells contain a varying number of AMPA receptors (AMPARs) per synapse that increases with distance from the soma, a phenomenon known as distancedependent scaling. Here, we undertake an investigation into the molecular mechanisms of distance-dependent scaling. Using dendritic recordings from rat pyramidal neurons, we confirm the basic scaling phenomenon and find that it is expressed and can be manipulated cell autonomously. Finally, we show that it depends on the presence of both a reserve pool of AMPARs and the AMPAR subunit GluA2.

\section{Introduction}

Information transfer between neurons, in the form of initiation or suppression of action potentials, occurs by the summing of many dendritic inputs in the soma. In this way, each individual neuron serves as a signal integrator, vastly increasing the information capacity of the system. However, as current travels along a dendrite, electrotonic filtering reduces the amplitude and slows the kinetics of electrical potentials. This represents a problem for the integration of signals in neurons with large, complex dendritic arbors whereby EPSPs from distal synapses would contribute less than EPSPs from proximal synapses. Indeed, this critically affects our understanding of neural circuits, in which synaptic capacity across the dendrites of each individual neuron is assumed to be homogeneous. Yet, in functioning hippocampal pyramidal neurons, the amplitude of somatic EPSPs has been shown to be independent of synapse distance from the soma, implying that a cellular compensation is occurring to counteract the dendritic filtering (Magee and Cook, 2000).

Converging evidence has implicated upregulation of synaptic AMPA receptors (AMPARs) in distal synapses as the reason that somatic EPSPs appear similar despite differing amounts of filtering (Magee and Cook, 2000; Andrasfalvy and Magee, 2001; Smith

Received Feb. 13, 2013; revised June 3, 2013; accepted June 29, 2013.

Author contributions: S.L.S. and R.A.N. designed research; S.L.S., B.E.H., and Y.H.S. performed research; S.L.S., Y.H.S., K.W.R., and R.A.N analyzed data; S.L.S. and R.A.N. wrote the paper.

This work was supported by a grant from the U.S. National Institute of Mental Health (R37 MH038256-29), and by the Intramural Research Program of National Institute of Neurological Disorders and Stroke (Y.H.S. and K.W.R.). We are grateful to Dan Johnston and the members of his laboratory, specifically Darrin Brager and Rishi Narayanan, for assistance with dendritic recording methodology.

The authors declare no competing financial interests.

Correspondence should be addressed to Roger A. Nicoll, Departments of Cellular and Molecular Pharmacology and Physiology, University of California San Francisco, 600 16th Street, Genentech Hall N272D, San Francisco, CA 94158-2517. E-mail: roger.nicoll@ucsf.edu.

DOI:10.1523/JNEUROSCI.0678-13.2013

Copyright $\odot 2013$ the authors $\quad 0270-6474 / 13 / 3313312-08 \$ 15.00 / 0$ et al., 2003), a phenomenon known as distance-dependent scaling. However, we know relatively little about how this scaling is achieved. Based on knock-out animals, it has been proposed that the scaling is responsive to activity (Andrásfalvy et al., 2008) and may even share mechanistic similarities with long-term potentiation (LTP) based on a requirement for the AMPAR subunit GluA1 (Andrásfalvy et al., 2003). The dependence on GluA1 differentiates LTP from another form of plasticity, synaptic homeostasis, which depends instead on GluA2 (Gainey et al., 2009; Goold and Nicoll, 2010). One might, then, pursue a model for distance-dependent scaling based on the molecules thought to be involved in LTP. However, it is not known whether the requirement for GluA1 is specific, as has been shown to be the case in LTP (Zamanillo et al., 1999; Hayashi et al., 2000; Shi et al., 2001), or might extend to other AMPAR subunits. Moreover, at the most basic level, it is not known whether distance-dependent scaling is generated by the network and greater architecture of the brain or, conversely, is a cell-autonomous phenomenon.

In this study, we set out to study the molecular properties of distance-dependent scaling. In doing so, we provide an independent confirmation of an increase in synaptic AMPAR content as a function of distance in CA1 pyramidal cells. We also show that the scaling is expressed, and may be manipulated, in a cellautonomous manner. Finally, we show that distance-dependent scaling requires both a reserve pool of AMPARs and specifically requires the GluA2 AMPAR subunit. Together, these results focus our understanding of distance-dependent scaling to the individual cell level and suggest mechanistic molecular similarities between this modulation of synaptic strength and diverse forms of synaptic plasticity.

\section{Materials and Methods}

Experimental constructs. Knockdown constructs targeting GluA2 (shGluA2, target sequence: ggagcactccttagcttga) and CNIH-2 (shCNIH-2, target sequence: gatgcggtctctatcatga) were expressed from 
an $\mathrm{H} 1$ promoter in pFHUGW along with GFP expressed from the Ubiquitin promoter to mark the transfected cell.

Lentivirus production. For the production of lentiviral particles, HEK293T cells were cotransfected with the expression construct (in pFHUGW) along with psPAX2 and pVSV-G using FuGENE HD (Roche). Forty hours later supernatant was collected, filtered, and concentrated by ultracentrifugation $\left(80,000 \mathrm{~g}\right.$ for $2 \mathrm{~h}$ at $\left.4^{\circ} \mathrm{C}\right)$ or precipitated using PEG-it (Systems Biosciences) and collected via centrifugation. The resulting viral pellet was resuspended in Opti-MEM and stored at $-80^{\circ} \mathrm{C}$ until use.

Primary neuron culture. Primary rat hippocampal neurons were prepared from embryonic day 18 Sprague Dawley rats (Harlan) following the guidelines of the National Institutes of Health Animal Research Advisory Committee (animal protocol number 1171). Briefly, hippocampi from rat embryos were isolated and incubated in chopping solution [HBSS (Invitrogen) with $10 \mathrm{~mm}$ HEPES, $1.37 \mathrm{mg} / \mathrm{ml}$ DNase (Sigma), and penicillin-streptomycin] with $0.05 \%$ trypsin (Sigma) for $12 \mathrm{~min}$ at $37^{\circ} \mathrm{C}$. Cells were washed three times with a cold chopping solution, and triturated 10-15 times with a fire-polished Pasteur pipette. The dissociated neurons were harvested by centrifugation at $160 \times \mathrm{g}$ for $3 \mathrm{~min}$ at $4^{\circ} \mathrm{C}$ and plated on poly-D-lysine (Sigma)-coated dishes in serum-free Neurobasal media (Invitrogen) with B-27 supplement and L-glutamine (Invitrogen).

Western blotting. Primary rat hippocampal neurons were infected with lentiviral particles at 7-10 days in vitro and maintained in culture for $7 \mathrm{~d}$. The neurons were harvested in TNE buffer ( $50 \mathrm{~mm}$ Tris-HCl, $\mathrm{pH} 8.0,150$ $\mathrm{mm} \mathrm{NaCl}, 2$ mм EDTA) containing protease inhibitor cocktail (Roche), briefly sonicated for $5 \mathrm{~s}$, and then centrifuged at 117,000 $\times g$ for $20 \mathrm{~min}$ at $4^{\circ} \mathrm{C}$. The pellets were resuspended in TNE buffer, and solubilized with $1 \%$ SDS for $5 \mathrm{~min}$ at room temperature. Finally, three to four volumes of TNE buffer with $1 \%$ Triton X-100 was added, followed by a $30 \mathrm{~min}$ incubation on ice. The lysates were centrifuged at $20,000 \times g$ for $15 \mathrm{~min}$ at $4^{\circ} \mathrm{C}$. The supernatants were mixed with $6 \times$ Laemmli buffer, resolved by SDS-PAGE, and transferred to PVDF membrane. The membranes were blocked in $0.1 \%$ TBS with $0.1 \%$ Tween 20 containing $5 \%$ skim milk, and then probed with the indicated primary antibodies. Anti- $\alpha$ tubulin antibody was obtained from Sigma, anti-CNIH-2 antibody was custommade (Herring et al., 2013), and all other antibodies were from Millipore. The blots were incubated with peroxidase-conjugated secondary antibodies and detected with ECL reagent (Thermo Scientific).

Stereotaxic injection. Concentrated lentiviruses were injected bilaterally into the medial hippocampi of isoflurane-anesthetized 4- to 5-weekold rats using a stereotaxic apparatus (Kopf). Lentivirus (500 nl per hemisphere) was delivered at $500 \mathrm{nl} / \mathrm{min}$ via a Hamilton (88011) syringe driven by a Micro4 microsyringe pump controller (WPI).

Acute slice preparation. Acute hippocampal slices were prepared from adult rats of either sex 10-12 d after virus injection. Rats were anesthetized through intraperitoneal injection of a ketamine/xylazine combination and transcardially perfused with a cold $\left(4^{\circ} \mathrm{C}\right)$ cutting solution $(210$ mu sucrose, $2.5 \mathrm{~mm} \mathrm{KCl}, 0.5 \mathrm{~mm} \mathrm{CaCl}_{2}, 7 \mathrm{mM} \mathrm{MgCl}_{2}, 1.25 \mathrm{~mm} \mathrm{NaH}_{2} \mathrm{PO}_{4}$, $25 \mathrm{~mm} \mathrm{NaHCO}_{3}, 7 \mathrm{~mm}$ glucose, $1.3 \mathrm{~mm}$ ascorbic acid, and $3 \mathrm{~mm}$ pyruvic acid saturated with $95 \% \mathrm{O}_{2} / 5 \% \mathrm{CO}_{2}$ ) before decapitation. While submerged in cutting solution, brains were removed and sliced into $350 \mu \mathrm{m}$ near-horizontal sections using a vibratome (DSK). Slices were then transferred to a holding chamber containing artificial CSF (ACSF) (125 mu NaCl, $2.5 \mathrm{~mm} \mathrm{KCl}, 1.25 \mathrm{~mm} \mathrm{NaH}_{2} \mathrm{PO}_{4}, 25 \mathrm{~mm} \mathrm{NaHCO}_{3}, 11 \mathrm{~mm}$ glucose, $1 \mathrm{~mm} \mathrm{MgSO}_{4}$, and $2 \mathrm{~mm} \mathrm{CaCl}_{2}$ saturated with $95 \% \mathrm{O}_{2} / 5 \% \mathrm{CO}_{2}$ ) and incubated for $30 \mathrm{~min}$ at $34^{\circ} \mathrm{C}$. These and all other experiments were performed in accordance with established protocols approved by the University of California San Francisco Institutional Animal Care and Use Committee.

Electrophysiological recording. Electrophysiological recordings were performed on an upright Olympus BX51WI microscope using a Multiclamp 700B amplifier (Molecular Devices). Acute slices were maintained in ACSF (125 mm NaCl, $2.5 \mathrm{~mm} \mathrm{KCl}, 1.25 \mathrm{~mm} \mathrm{NaH}{ }_{2} \mathrm{PO}_{4}, 25 \mathrm{~mm}$ $\mathrm{NaHCO}_{3}$, and $11 \mathrm{~mm}$ glucose saturated with $95 \% \mathrm{O}_{2} / 5 \% \mathrm{CO}_{2}$ ) containing $1 \mathrm{~mm} \mathrm{MgSO}_{4}$ and $2 \mathrm{~mm} \mathrm{CaCl}_{2}$. Dendritic recordings were obtained using thick-walled glass recording pipettes containing the following (in mM): $120 \mathrm{KMeSO}_{3}, 20 \mathrm{KCl}, 10 \mathrm{HEPES}, 4 \mathrm{NaCl}, 0.5 \mathrm{EGTA}, 0.3 \mathrm{CaCl}_{2}, 4$ Mg-ATP, 0.3 Na-GFP, and 5 QX-314. Miniature EPSCs (mEPSCs) were isolated by the addition of $1 \mu \mathrm{M}$ tetrodotoxin (TTX) and $100 \mu \mathrm{M}$ picro- toxin to the ACSF. Other than the double-patch experiments presented in Figure 2, individual dendrites were only patched once, at a single location. No drugs were present during double-patch experiments (Fig. 2). Lucifer yellow was added to the internal solution to allow tracing of the dendrite from the recording location to the cell body for measurements of distance. Pipette resistances for dendritic recordings were between 5 and $8 \mathrm{M} \Omega$, input resistances ranged between 100 and $400 \mathrm{M} \Omega$, and series resistances were between 15 and $45 \mathrm{M} \Omega$. We found no bias in series resistance with respect to dendritic location, decay kinetics, or rise kinetics. Hyperosmotic solution to elicit local mEPSCs was composed of ACSF with the addition of $300 \mathrm{~mm}$ sucrose and $0.5 \mu \mathrm{M}$ TTX and was puffed locally via a patch pipette. To assess rectification, simultaneous dual whole-cell recordings were performed using an internal solution containing the following (in mM): $135 \mathrm{CsMeSO}_{3}, 10 \mathrm{HEPES}, 8 \mathrm{NaCl}, 0.3$ EGTA, 4 Mg-ATP, $0.3 \mathrm{Na}-\mathrm{GTP}$, 5 QX-314, and 0.1 spermine, with the addition of $50 \mu \mathrm{M}$ APV to the ACSF to block NMDA receptors (NMDARs). To evoke synaptic responses, a bipolar stimulating electrode was placed in the Schaffer collateral pathway. Somatic outside-out patches were excised from CAl pyramidal neurons using 3-5 M $\Omega$ pipettes containing the same internal solution used to assess rectification. Outside-out patches were held at $-70 \mathrm{mV}$ and were continually perfused with ACSF additionally containing $0.1 \mathrm{~mm}$ cyclothiazide, $100 \mu \mathrm{M}$ D-APV, and $0.5 \mu \mathrm{M}$ TTX. AMPAR-mediated currents in outside-out patches were elicited with a $2 \mathrm{~s}$ pulse of $1 \mathrm{~mm}$ glutamate in ACSF. Recordings were filtered using a $2 \mathrm{kHz}$ Bessel filter and digitized at $10 \mathrm{kHz}$. mEPSCs with amplitudes $\geq 4 \mathrm{pA}$ and a rates of rise $\geq 4 \mathrm{pA} / \mathrm{ms}$ were automatically detected and analyzed off-line with customized software (Igor). Rectification index was calculated as the ratio of scaled (multiplied by 1.75) current at $+40 \mathrm{mV}$ to current at $-70 \mathrm{mV}$.

Statistical analysis. Between-set comparisons of binned data (e.g., proximal vs distal, wild-type vs knockdown) were analyzed for statistical significance using a Mann-Whitney $U$ test. Probability distributions were analyzed using a Kolmogorov-Smirnov test. Regression statistics in all cases reflect analysis by a linear fit. The reported $n$ indicates the number of individual recordings.

\section{Results}

\section{Verification of a distance-dependent synaptic AMPAR gradient in CA1 pyramidal neurons}

Given the fundamental nature of distance-dependent scaling to our understanding of electrical integration within pyramidal cells, we first set out to measure the strength of AMPAR-mediated synaptic responses as a function of distance from the soma in CA1 pyramidal neurons in an effort to independently confirm the existence of a distance-dependent gradient. Unfortunately, accurate measurements of the local synaptic currents cannot be made from the cell's soma where a cell is typically patched; passive electrical filtering and the difficulty of achieving voltage clamp over distance contribute too heavily to the recording of currents that originate far from the site of the patch pipette (Williams and Mitchell, 2008). For accurate estimations of AMPAR content at a synapse, the recording pipette must be as physically close to the synapses of interest as possible. Therefore, we recorded miniature AMPAR EPSCs (mEPSCs) by directly patching the primary apical dendrite of hippocampal CA1 pyramidal neurons while locally stimulating the release of presynaptic vesicles of neurotransmitter with puffs of hyperosmotic solution (Fig. 1A). Since the rate of spontaneous mEPSCs in this preparation is very low (Fig. $1 A$, black trace), these recordings contain a nearly pure population of $\mathrm{MEPSCs}$ arising from the area just around the recording pipette, the amplitude of which serves as a readout for local synaptic AMPAR content. By varying the distance of the recordings from the cell body of the neuron, we were able to measure the amplitude of mEPSCs as a function of distance along the apical length of the dendrite.

We found that mEPSC amplitudes increase with distance from the soma (Fig. 1B), in perfect agreement with Magee et al. 

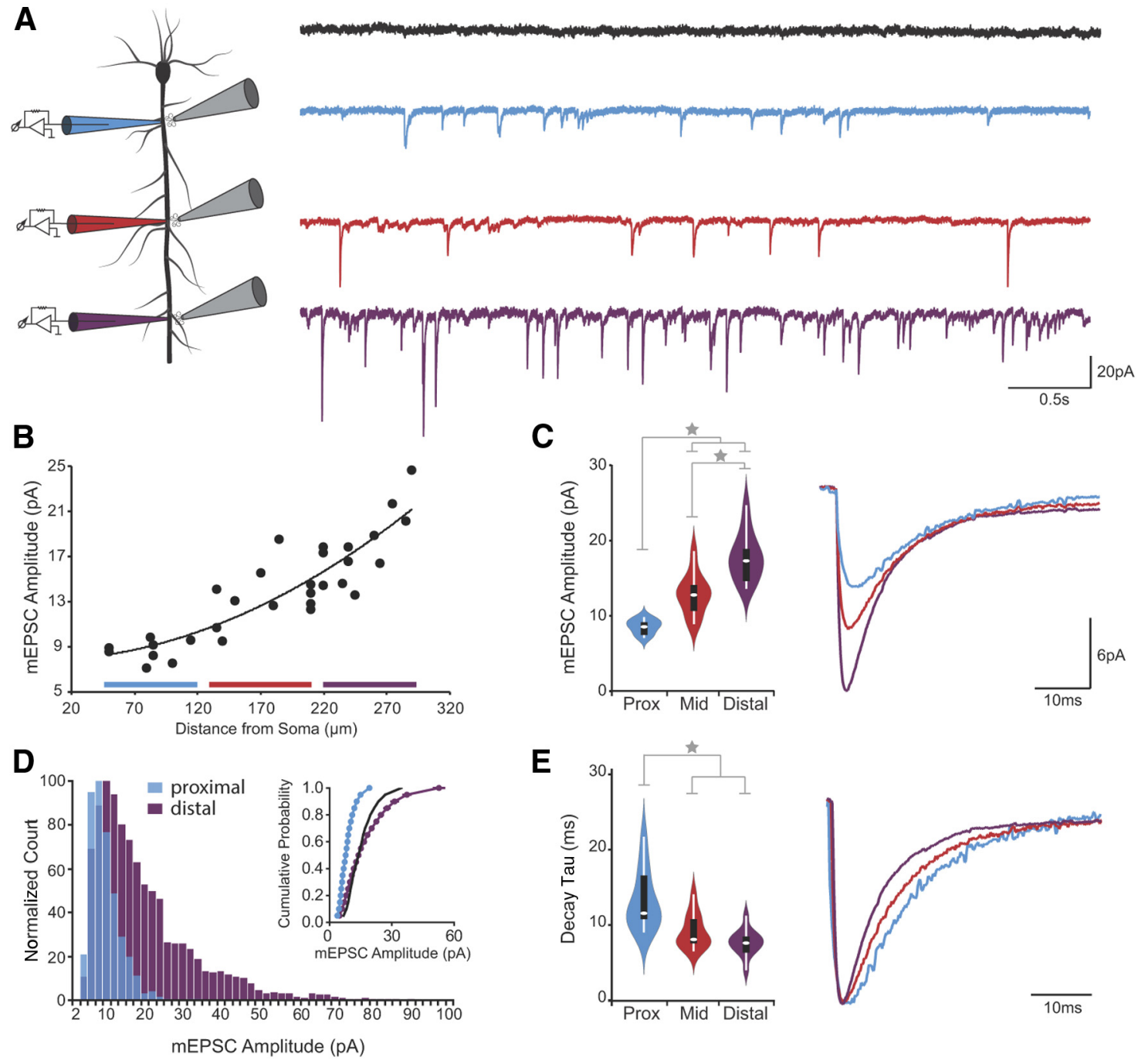

Figure 1. Distance-dependent scaling of AMPARs. A, Schematic of the recording setup and example traces. CA1 pyramidal neurons were patched at varying distances along their primary apical dendrites and $\mathrm{mEPSC}$ s were recorded after being locally elicited by the application of hyperosmotic solution. Black, top trace shows the lack of spontaneous activity in the absence of hyperosmotic solution at a proximal location. Bottom traces show representative currents recorded from proximal (blue), mid (red), and distal (purple) locations. $\boldsymbol{B}$, mEPSC amplitude increases as a function of distance from soma ( $n=31$; linear fit has nonzero slope, $\left.p<0.0001, R^{2}=0.7617\right)$. Filled circles are individual recordings; line is a second-order polynomial regression. $C$, Average $\mathrm{mEPSC}$ amplitudes binned by location; proximal ( $50-120 \mu \mathrm{m})$, $\operatorname{mid}(130-210 \mu \mathrm{m})$, and distal $(220-290 \mu \mathrm{m})$. Compared with the amplitudes of proximal events $(n=8)$, both mid $(n=11 ; p<0.005)$ and distal ( $n=12 ; p<0.005) \mathrm{mEPSCs}$ are larger. Distal mEPSCs are additionally larger than mid ( $p<0.0005)$. Violin plot shows median (white oval), interquartile range (black bar), range (white line), and a probability density function to indicate the distribution of the data. Traces to the right show average proximal (blue), mid (red), and distal (purple) $\mathrm{mEPSCS}$. D, Histogram of mEPSC amplitudes for all proximal and distal events showing an augmentation of large amplitude distal mEPSCs. Inset shows cumulative probability of mEPSC amplitudes (mean $\pm S E M$ ). Black line shows proximal amplitudes scaled by 1.8. By Kolmogorov-Smirnov comparison, the distribution of distal events differs from both the proximal $(p<0.0001)$ and scaled-proximal distributions $(p<$ $0.0001)$. $E$, Weighted tau for the decay of mEPSCs binned by location as in C. Compared with the decay of proximal events, both mid $(p<0.05)$ and distal $(p<0.001) \mathrm{mEPSCs}$ are faster. Mid and distal decay times are not significantly different $(p>0.05)$. Violin plot is analogous to that in $C$. Traces to the right show average mEPSCs as in $C$, scaled and aligned to their peaks.

(2000). To be specific, the average amplitude of the most distal synapses in stratum radiatum was slightly more than double that of the most proximal synapses (Fig. 1C). By comparing the histogram of synaptic amplitudes obtained with distal and proximal recordings, it becomes clear that the distal increase in amplitude is primarily due to an augmentation of large amplitude responses with a very slight change in the distribution of lower amplitude events (Fig. 1D). The original electrophysiological characterization of the scaling phenomenon found a similar preservation of small amplitude events at distal locations (Magee and Cook, 2000), although this was not found to be the case in a subsequent study (Smith et al., 2003). This distinction has a profound implication on the interpretation of the phenomenon as the data presented here do not represent scaling of the response amplitude in the sense orig- inally applied to describe synaptic homeostasis (Turrigiano et al., 1998); that is, a multiplicative scaling factor applied uniformly as a function of initial (or in this case proximal) amplitude (Fig. $1 D$, inset).

Similar to the original characterization of distancedependent scaling, we also recorded an increase in the decay rate of AMPAR mEPSCs along the proximal distal axis (Fig. $1 E)$. This speeding of distal currents may be largely accounted for by properties of the membrane (Magee and Cook, 2000) and voltage-gated conductances that serve to normalize the temporal integration of synaptic inputs over the dendritic arbor (Magee, 1999). The result of the initial investigation of AMPAR-mediated synaptic currents as a function of distance, then, confirms the existence of the phenomenon of distancedependent scaling. 
A

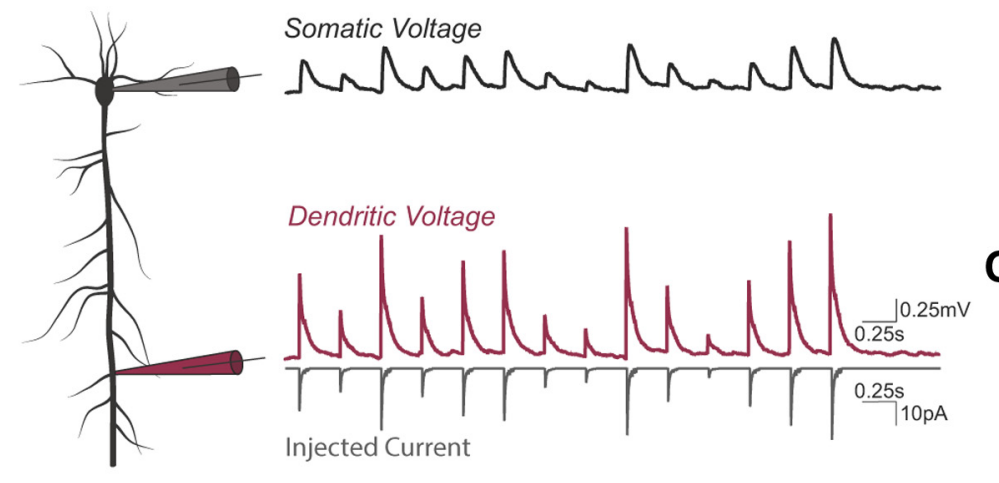

B
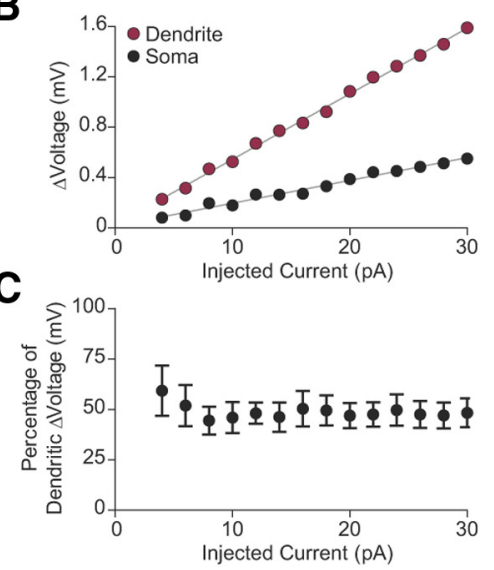

Figure 2. Dendritic filtering as a function of input amplitude. $\boldsymbol{A}$, Schematic of the recording setup and example traces. A single CA1 pyramidal neuron was patched simultaneously at somatic and dendritic (mid-distal) locations. Voltage is monitored at both locations in current clamp while mEPSC-shaped currents of varying amplitudes are injected at the distal site. Black, top trace shows a representative recording from a somatic location while the lower, red trace shows a simultaneous recording from a dendritic location. Bottom, gray trace shows the injected current. $\boldsymbol{B}$, Change in voltage as a function of the injected current amplitude recorded simultaneously at dendritic (in red) and somatic (in black) sites for a representative experiment. $\boldsymbol{C}$, Average (mean \pm SEM, $n=5$ ) amplitude filtering, somatic voltage change as a percentage of the local dendritic voltage change, in response to a range of injected current amplitudes. Uniform filtering is evident without regard to input amplitude.

\section{The filtering of dendritic potentials as a function of amplitude}

Given that the distance-dependent increase in AMPAR currents at distal synapses did not represent a pure multiplicative increase in the distribution of mEPSC current amplitudes seen at proximal synapses, we wondered whether there may exist a nonlinearity in the filtering of dendritic potentials as a function of their initial amplitude. That is, perhaps larger synaptic currents would be filtered to a greater or lesser extent as they travel toward the soma than smaller synaptic currents, due to the activation of voltage-gated conductances. If dendritic potentials are filtered differently as a function of their initial size, one would not expect the distance-dependent increase to follow strictly multiplicative scaling to normalize input strength as measured at the soma. We directly tested whether dendritic potentials are filtered differentially as a function of their input amplitude by double patching the same cell, at the soma and the dendrite, in the absence of any pharmacology to block voltage-gated conductances. We then injected mEPSC-shaped currents of varying amplitudes into the dendritic site while monitoring voltage at both locations (Fig. $2 A$ ). As expected, we found substantial filtering of the potentials when recorded at the soma rather than locally in the dendrite (Fig. 2B). However, no obvious bias was seen in the amount of filtering as a function of the injected current amplitude (Fig. 2C), indicating that, at least over the range of amplitudes tested, the filtering that occurs as current travels along the dendrite is linear with respect to initial amplitude.

\section{Evidence for cell-autonomous scaling of AMPAR currents}

To understand the molecular basis of distance-dependent scaling, it would be beneficial to first know whether the gradient is regulated on a cell-by-cell basis or, rather, is dependent on cell-extrinsic factors-those diffusible extracellular signals that participate in the organization of the circuit in the first place. The stereotyped architecture of the hippocampus lends considerable ambiguity to this question: although the gradient of AMPARs is expressed as a function of distance from the cell body, the tight arrangement of the cell bodies of all CA1 pyramidal neurons into a single layer means that the gradient is also expressed within the coordinates of the larger structure of the hippocampus. So then, how can we know whether the AMPAR gradient is referenced to the cell body (and likely cell autonomous) or, rather, the cell layer (and likely influenced by factors extrinsic to the pyramidal neuron)? To answer this question, we left behind the typical CA1 pyramidal neuron and turned to a relatively unstudied cell type of the hippocampus: the pyramidal-like principal neuron, or PLP cell. The gross morphology of a PLP cell is quite similar to that of an ordinary CA1 pyramidal neuron, but the location of the cell body is perfectly suited to the question at hand. Rather than having their cell bodies tightly packed within the cell layer, PLP cells are scattered throughout stratum radiatum; this distribution, in fact, is their primary identifying feature (Fig. $3 A$, blue cells) (Bullis et al., 2007). The orientation of their apical dendrite runs parallel to that of the layer-based pyramidal neurons, but the location of individual PLP cell bodies varies within the larger architecture of the hippocampus. Assuming that these PLP cells also display distance-dependent scaling, there are two possible scenarios: one in which the scaling will be referenced to the cell body, indicating a cell-autonomous mechanism, and another in which the scaling will be referenced to the architecture of the hippocampus, the cell layer in particular, indicating an external cue.

As we did for the CA1 pyramidal neurons, we recorded local mEPSCs via dendritic patches along the length of the primary apical dendrite of PLP cells. We then analyzed these currents as a function of both distance from the cell soma and as a function of distance from the cell layer. The mEPSC amplitudes recorded from PLP cells display a distance-dependent gradient that closely matches that of layer pyramidal neurons when referenced to the soma (Fig. 3B), but does not match when referenced to the cell layer (Fig. 3C). This soma-referenced gradient strongly suggests that distance-dependent scaling is a cell-autonomous feature, rather than a consequence of the hippocampal architecture or diffusible molecular cues.

A sufficient supply of AMPARs is necessary for the expression of distance-dependent scaling

If the distance-dependent scaling of AMPARs is cell autonomous, then one should be able to influence it using molecular manipulations of an individual cell. Previously it was shown that a mouse 

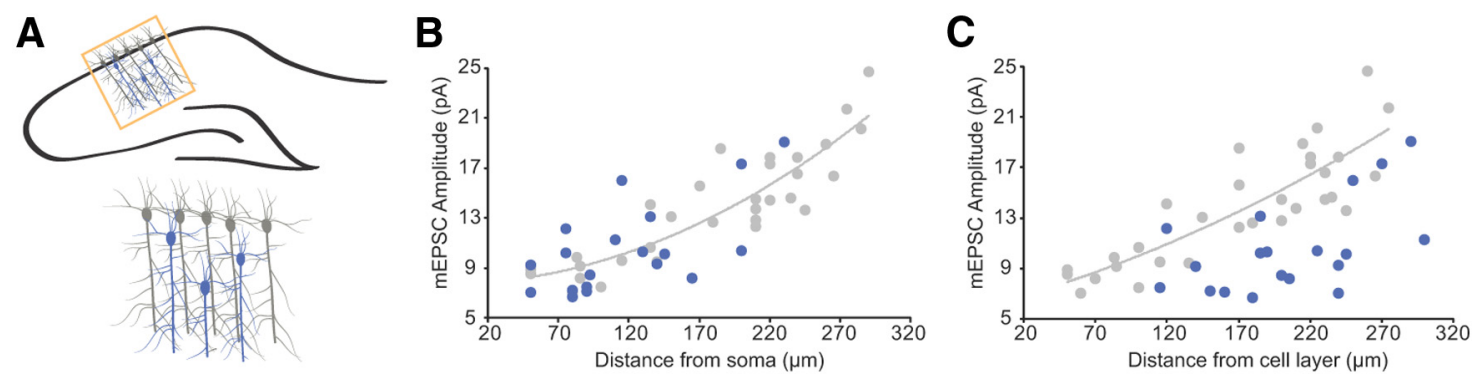

Figure 3. AMPAR gradient is referenced to the soma in PLP cells. A, Organization of PLP cells. As opposed to CA1 pyramidal neurons (in gray) that have their cell bodies tightly packed in a layer, PLP cells (in blue) have their cell bodies scattered throughout stratum radiatum. $\boldsymbol{B}$, When referenced to the soma, AMPAR mEPSCs recorded from PLP cells increase with distance and match the scaling curve found in CA1 pyramidal neurons ( $n=19$; linear fit has nonzero slope, $p<0.005, R^{2}=0.4184$ ). This linear fit of PLP amplitudes does not differ from that of CA1 pyramidal neuron amplitudes in either slope $(p>0.05)$ or elevation $(p>0.05)$. Blue filled circles show individual PLP recordings. CA1 pyramidal recordings (originally shown in Fig. 1B) are repeated here as gray filled circles for comparison. $\boldsymbol{C}$, When referenced to the cell layer, PLP mEPSCs amplitudes do not match those recorded from CA1 pyramidal neurons (difference in elevation, $p<0.0001$ ). Points are analogous to those in $\boldsymbol{B}$.

lacking the AMPAR subunit GluA1-AMPARs in CA1 pyramidal neurons being heterotetramers containing GluA1, 2, or 3 (Wenthold et al., 1996)-does not express distance-dependent scaling (Andrásfalvy et al., 2003). AMPAR-mediated currents in this mouse were normal at proximal synapses, but failed to increase as a function of distance. The interpretation of this result was that distance-dependent scaling may share a molecular pathway with a form of synaptic plasticity, LTP, which has also been shown to be dependent on GluA1. However, recent work has challenged the dependence of LTP on the GluA1 subunit, instead arguing that loss of GluA1 simply depletes a reserve pool of surface AMPARs that are necessary for the expression of LTP, independent of subunit composition (Granger et al., 2013).

Another method to deplete this reserve pool of AMPARs is to eliminate the AMPAR accessory subunit cornichon-2 (CNIH-2), which is involved in the surface trafficking of AMPARs (Schwenk et al., 2009; Shi et al., 2010; Herring et al., 2013). We therefore made use of an shRNA targeting CNIH-2 (Fig. $4 A$ ), which, like the deletion of GluA1, reduces the surface pool of AMPARs (Herring et al., 2013). We expressed this shRNA from a lentivirus, which was injected into the hippocampi of 4-week-old rats. This results in a very sparse distribution of shRNA-expressing pyramidal cells in CA1. Ten to 12 d later, we identified transduced cells via fluorescence and locally recorded sucrose-evoked mEPSCs from these cells as previously described. When the amplitudes of these currents are examined as a function of distance from the soma, it is clear that the knockdown of CNIH-2 in individual cells results in a similar loss of distance-dependent scaling expression as was seen in the GluA1 knock-out (Fig. $4 B, C$ ); a result that confirms that this phenomenon may be manipulated in a cellautonomous manner and is consistent with dependence on a pool of reserve AMPARs.

CNIH-2 has also been shown to influence the kinetics of the AMPAR response. Specifically, its presence has been shown to slow the kinetics of an AMPAR (Schwenk et al., 2009; Shi et al., 2010; Gill et al., 2011; Herring et al., 2013). We speculated that a theoretical gradient of CNIH-2 could partially explain the faster kinetics of distal mEPSCs. Knockdown of CNIH-2 did, as expected, speed mEPSC kinetics (Fig. 4D,E). However, both the proximal and distal mEPSCs became faster, thus preserving the increase in mEPSC decay over the length of the dendrite (Fig. $4 D, E)$. A theoretical CNIH-2 gradient, therefore, does not explain the observed kinetic gradient in wild-type cells.

\section{Distance-dependent scaling additionally requires the GluA2 subunit}

Loss of the reserve pool of AMPARs results in a loss of both LTP and distance-dependent scaling as evidenced by the GluA1 knock-out and now a CNIH-2 knockdown. Thus, there are some molecular similarities between distance-dependent scaling and at least one form of synaptic plasticity, LTP. Another form of synaptic plasticity, occurring over much longer timescales, is synaptic homeostasis-the alteration of synaptic strength in response to long-term changes in activity level of a neuron. This form of synaptic plasticity has been shown to depend on a different AMPAR subunit: GluA2 (Gainey et al., 2009; Goold and Nicoll, 2010). Loss of GluA2 affects the neuron in a much different way than loss of GluA1. Whereas loss of GluA1 greatly depletes both synaptic and extrasynaptic AMPARs, loss of GluA2 only depletes synaptic receptors (Lu et al., 2009). Thus, LTP is normal in GluA2 knock-out cells (Meng et al., 2003) because the reserve pool of AMPARs remains intact (Granger et al., 2013). The dependence of synaptic homeostasis on GluA2, on the other hand, has been ascribed to something more intrinsic to the GluA2 subunit rather than simply a pool of receptors.

Might we be able to distinguish distance-dependent scaling from these divergent forms of plasticity based on its subunit dependence? To answer that question, we used an shRNA targeting GluA2, expressed via lentivirus and injected into the hippocampi of 4-week-old rats as before, to assess distance-dependent scaling in the absence of GluA2. This shRNA was validated by Western blotting (Fig. $5 A$ ) and functionally via rectification in neurons (Fig. 5B). Specifically, we compared rectification of evoked synaptic currents in control neurons to rectification in neurons expressing the shRNA against GluA2 and found that the control neurons had linear currents (GluA2-containing), while those expressing the shRNA displayed nearly complete rectification (GluA2-lacking) (Fig. 5B). Importantly, conditional removal of GluA2, unlike the removal of GluA1 or CNIH-2, does not alter the surface expression of AMPARs (Lu et al., 2009; Herring et al., 2013). We confirmed that the knockdown of GluA2 similarly does not reduce the surface pool of AMPARs as assessed by outside-out patch recordings from wild-type and shRNAexpressing cells (Fig. 5C).

When we examined mEPSCs in cells expressing shGluA2 as a function of distance, we found that normal distance-dependent scaling was absent (Fig. 5D). Surprisingly, we actually found that knockdown of GluA2 lead to a slight reversal of the AMPAR 
A

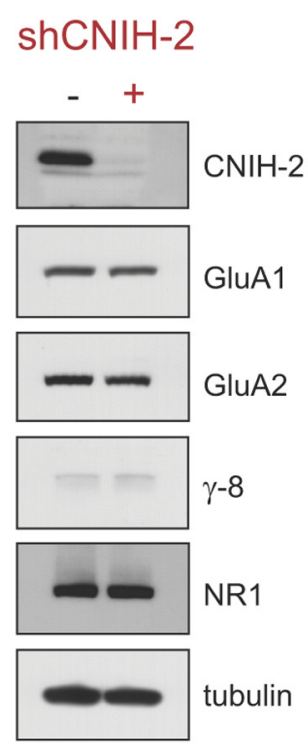

B

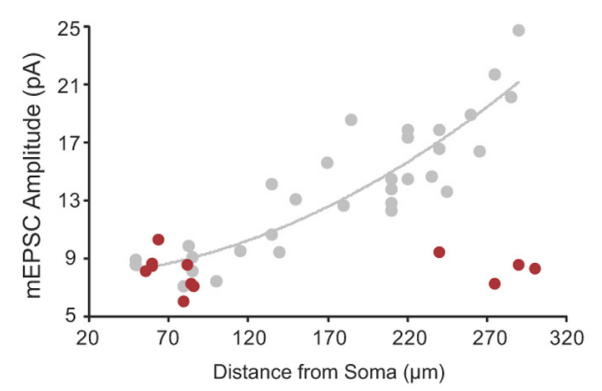

D

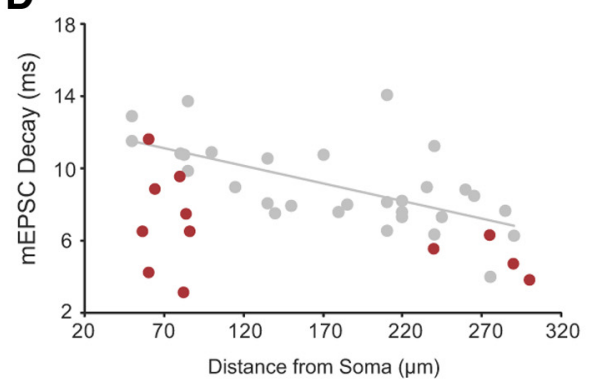

C

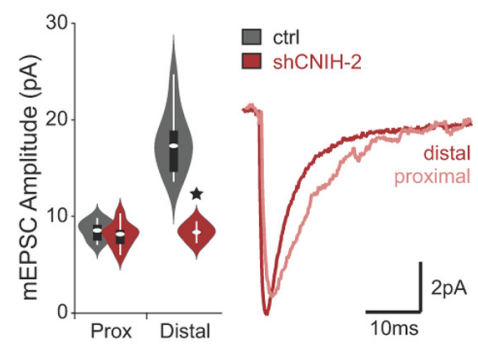

E

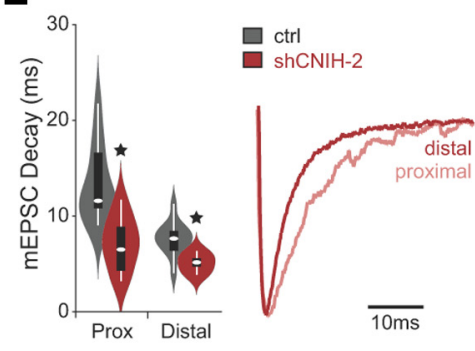

Figure 4. Cornichon-2 knockdown selectively reduces distal EPSCS. A, Lentiviral expression of an shRNA targeting CNIH-2 in dissociated neurons drastically reduces CNIH-2 protein without affecting the abundance of other synaptic proteins. $\boldsymbol{B}$, Following knockdown of CNIH-2, AMPAR mEPSCs recorded from CA1 pyramidal cells do not increase as a function of distance from the soma ( $n=12$, slope of linear fit differs from control, $p<0.0001)$. Red filled circles show individual recordings from shCNIH-2-expressing cells, gray filled circles show recordings from control cells (originally shown in Fig. 1B) for comparison. C, Average mEPSC amplitudes binned by location: proximal ( $50-120 \mu \mathrm{m})$ and distal ( $220-290 \mu \mathrm{m})$ with respect to CA1 pyramidal soma location. Proximal mEPSC amplitudes following knockdown $(n=8)$ do not differ from control proximal amplitudes $(p>0.05)$, whereas distal mEPSC amplitudes following knockdown $(n=4)$ are $\mathrm{smaller}$ than control distal amplitudes $(p<0.005)$. No difference exists between proximal and distal mEPSC amplitudes following knockdown $(p>0.05)$. Violin plot shows median (white oval), interquartile range (black bar), range (white line), and a probability density function to indicate the distribution of the data. Control plots are repeated from Figure 1 ( for comparison. Traces to the right show average proximal (light red) and distal (dark red) mEPSCs. D, The decay kinetics of mEPSCs speed as a function of distance from the soma in control cells ( $n=31$; linear fit has nonzero slope, $\left.p<0.0005, R^{2}=0.3836\right)$. This increase in kinetics as a function of distance remains after knockdown of CNIH-2 ( $n=12$; slope of linear fit does not differ from control, $\left.p>0.05\right)$, although there is a global speeding of decay (elevation of fit differs from control, $p<0.0001$ ). Points are analogous to those in $\boldsymbol{B}$. E, Weighted tau for the decay of mEPSCs binned by location as in $\boldsymbol{C}$. Compared with the decay of control mEPSCs, shCNIH-2-expressing cells have faster proximal $(p<0.01)$ and distal $(p<0.05)$ events. Violin plot is analogous to that in $C$. Control plots are repeated from Figure $1 E$ for comparison. Traces to the right show average $m E P S C s$ as in $C$, scaled and aligned to their peaks.

gradient (Fig. 5D,E). Thus, distance-dependent scaling requires the GluA2 subunit and presumably shares molecular pathway components with synaptic homeostasis. Interestingly, the distance-dependent increase in the kinetics of synaptic response was unaffected by knockdown of GluA2 (Fig. $5 F, G$ ).

\section{Discussion}

This study provides important insight into the molecular mechanisms of distance-dependent scaling. Specifically, we provide evidence that distance-dependent scaling of synaptic AMPAR content is expressed cell autonomously rather than being downstream of extracellular factors or network activity. Additionally, distance-dependent scaling can be disrupted on a cell-by-cell basis with knockdown of either CNIH-2 or GluA2. The dependence on CNIH-2 suggests a reliance on a reserve pool of AMPARs, whereas the dependence on GluA2 suggests molecular similarity to homeostatic synaptic plasticity.

The evidence for cell-autonomous expression of distancedependent scaling comes primarily from the finding that AMPAR-mediated currents in PLP cells appear to scale with respect to the location of their soma rather than the larger architecture of the hippocampus. It is worth noting, however, that PLP cells, although excitatory and pyramidal, are not identical to layer-based pyramidal cells. For instance, there is evidence that PLP cells express a reversed gradient of $I_{h}$ along the proximal distal axis of their apical dendrite (Bullis et al., 2007). Moreover, our current findings that distance-dependent scaling can be ma- nipulated in a cell-autonomous manner do not directly confirm a cell-autonomous expression mechanism. There exists the formal possibility that distance-dependent scaling operates through a combination of cell-autonomous and extrinsic cues.

It is debatable whether this distance-dependent increase in AMPAR content really represents a pure scaling of responses. The concept of synaptic scaling, as it has been applied to the homeostatic control of synaptic strength, has the defining principle of affecting all synapses in proportion to their initial strength (Turrigiano et al., 1998). Such scaling is therefore achieved by applying a multiplicative or divisive factor to all synapses equally, which is critical for maintaining the relationship of synapses to preserve information content of the system (Turrigiano, 2008). Based on the data presented here, the increase in distal mEPSC amplitudes is not the result of applying a multiplicative factor across all proximal amplitudes (Fig. 1D). Rather, at distal locations, there is a selective increase in large amplitude events with very little change in the distribution of small amplitude events. This is consistent with a previous report that showed, using immunogold electron microscopy, a selective increase in the number and density of AMPARs in large, perforated synapses in distal stratum radiatum without a change in the AMPAR composition of smaller synapses (Nicholson et al., 2006).

Given that the amplitude of somatically recorded EPSPs has been empirically shown to be independent of synapse location (Magee and Cook, 2000), there are two possible explanations for 
the skewing of mEPSC amplitude distributions that we observe at distal locations. First, it could be that the properties of the membrane and the presence of voltagegated conductances affect the propagation of synaptic currents differently depending on their initial amplitude. Large amplitude responses may engage more voltage-gated channels than small amplitude responses, some of which actively oppose depolarization of the cell $\left(I_{A}, I_{h}\right.$, and $I_{M}$, for example). If, as a result, large amplitude currents are filtered more strongly than small amplitude currents as they travel along a dendrite, then large amplitude synapses would need to be increased in strength as a function of distance by a larger factor than that of small amplitude synapses to normalize the contribution to somatic depolarization. However, we found no evidence for differential filtering of large amplitude currents, suggesting that this explanation is unlikely to account for the non-multiplicative scaling.

On the other hand, the skewing of the distribution of mEPSC amplitudes at distal locations could reflect a fundamentally different integration model than the one that is currently accepted. Distal dendrites could, in fact, possess two separate populations of synapses: locally acting, small amplitude synapses, and globally acting, large amplitude synapses. Perhaps there is a population of distal synapses that do represent a pure multiplicative scaling of proximal synapse amplitudes, and those synapses are responsible for the somatic depolarizations whose amplitudes are independent of synapse location. There may then be an additional population of distal synapses that have small amplitudes that are not strong enough to contribute individually to depolarization of the soma, but do contribute to local depolarizations. These small amplitude synapses would sum in a way that is conceptually similar to the two-stage model of dendritic integration (Katz et al., 2009), whereas the large amplitude synapses would follow a global integration model. Distinguishing between these two possible scenarios will be an exciting avenue of further experimentation.

When using RNAi, it is always prudent to consider the potential for off-target effects. At the protein level, we were able to show that a number of other synaptic proteins are unchanged following knockdown of our target RNAs. However, due to limitations of the system that we used-including viral delivery of experimental constructs, which has a restriction in the size of transcripts that can be delivered, and rat as our model system, which precludes the use of knock-outs-we were unable to directly assess the potential influence of off-target effects by

F
B

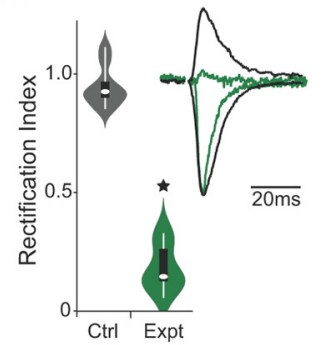

C

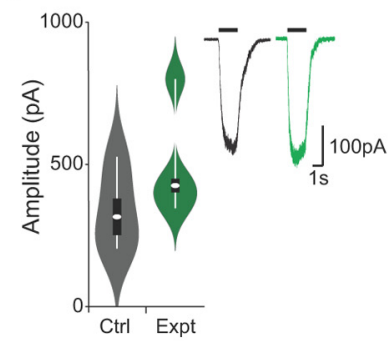

E
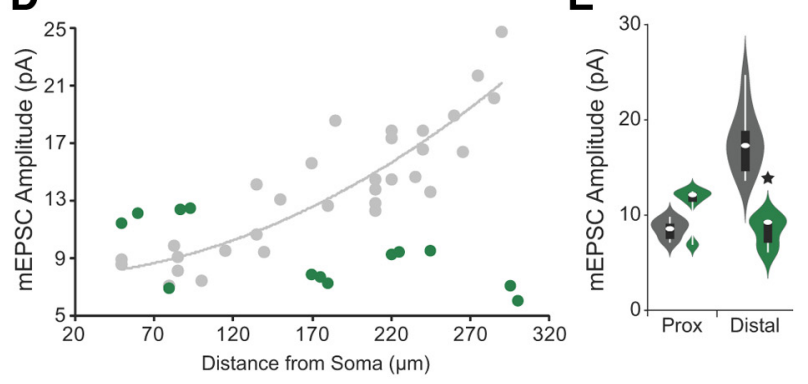

ctrl

shGluA2

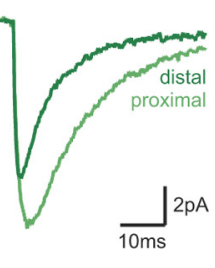

G
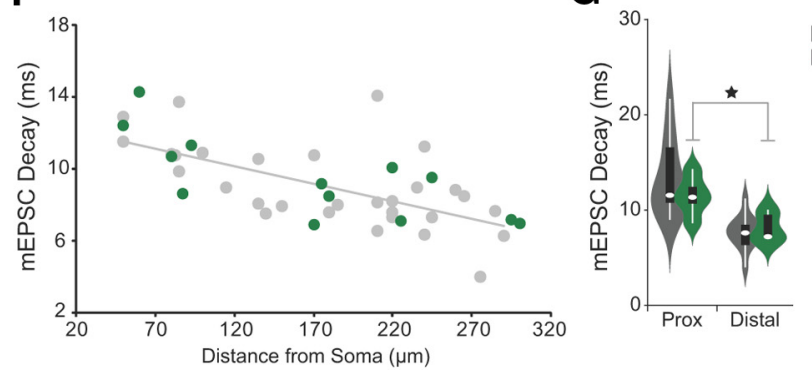

$\square$ ctrl

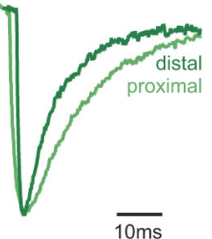

Figure 5. GluA2 knockdown reverses the distance-dependent scaling of AMPARs. $\boldsymbol{A}$, Lentiviral expression of an shRNA targeting GluA2 in dissociated neurons drastically reduces GluA2 protein without affecting the abundance of other synaptic proteins. $\boldsymbol{B}$, Expression of the shRNA in CA1 pyramidal neurons results in inwardly rectifying AMPAR-mediated evoked currents (rectification index is the ratio of current at $+40 \mathrm{mV}$ to current at $-70 \mathrm{mV}$, scaled by driving force) consistent with a loss of GluA2 ( $n=9 \mathrm{Ctrl}$, 9 Expt; $p<0.0001$ ). Traces show representative currents at $-70 \mathrm{mV}$ and $+40 \mathrm{mV}$ for control (in black) and experimental (in green) cells. Violin plot shows median (white oval), interquartile range (black bar), range (white line), and a probability density function to indicate the distribution of the data. C, Knockdown of GluA2 does not alter the surface expression of AMPARs at the soma of CA1 pyramidal cells as assessed by outside-out patch currents $(n=4, p>0.05)$. Traces show representative currents from control (in black) and experimental (in green) cells in response to a brief application of glutamate (indicated by black bar). Violin plot is analogous to that in $\boldsymbol{B}$. D. Following knockdown of GluA2 in CA1 pyramidal neurons, AMPAR-mediated mEPSCs no longer increase, but rather decrease as a function of distance from the soma $(n=13$; linear fit has nonzero, negative slope, $p<0.05$, $\left.R^{2}=0.4264\right)$. Green filled circles show individual recordings from shGluA2-expressing cells, gray filled circles show recordings from control cells (originally shown in Fig. 1B) for comparison. $\boldsymbol{E}$, Average mEPSC amplitudes binned by location: proximal (50-120 $\mu \mathrm{m})$ and distal $(220-290 \mu \mathrm{m})$. Distal mEPSC amplitudes following knockdown are reduced compared with control amplitudes $(n=5, p<0.005)$, while there is a trend toward increased proximal amplitudes following $\operatorname{knockdown}(n=5, p=0.0932)$. Violin plot is analogous to that in $\boldsymbol{B}$. Control plots are repeated from Figure 1 C for comparison. Traces to the right show average proximal (light green) and distal (dark green) mEPSCs. $\boldsymbol{F}$, Knockdown of GluA2 does not affect the speeding of AMPAR-mediated currents as a function of distance ( $n=13$; linear fit has nonzero slope, $p<0.005, R^{2}=0.5598$ ) with no change in the slope $(p>0.05)$ or elevation $(p>0.05$ ) compared with control. Points are analogous to those in $\boldsymbol{D}$. Control points are repeated from Figure $4 D$ for comparison. $\boldsymbol{G}$, Weighted tau for the decay of mEPSCs binned by location as in $\boldsymbol{E}$. Following knockdown of GluA2, distal mEPSCS decay faster than proximal mEPSCs $(p<0.05)$ with no change in decay kinetics for proximal $(p>0.05)$ or distal $(p>0.05)$ events compared with control. Violin plot is analogous to that in $\boldsymbol{B}$. Control plot is repeated from Figure $1 E$ for comparison. Traces to the right show average mEPSCs as in $\boldsymbol{E}$, scaled and aligned to their peaks. molecular rescue or expression in a knock-out background. This is not to say that we chose our system poorly. We simply prioritized other features such as the ability to express our constructs sparsely at a time when the development of the brain is nearly complete and the increased stability of dendritic recordings in a larger rodent. As the toolbox of molecular genetics expands, future experiments may be designed to alleviate the potential caveats introduced by the use of RNAi. 
The reliance of distance-dependent scaling on GluA2 expression suggests a molecular similarity to synaptic homeostasis. How might this guide future research into this phenomenon? GluA2 has been suggested to function relatively late in the molecular pathway of synaptic homeostasis (Goold and Nicoll, 2010). Upstream of GluA2, homeostasis has been shown to occur as a consequence of calcium entry through voltage-dependent calcium channels (Turrigiano, 2008), which functions as a signal of neural activity by binding calmodulin and activating CaM kinases (Wayman et al., 2008). Backpropagating action potentials may also serve as an instructive signal for synapse distance by generating a calcium gradient through voltage-dependent calcium channels and NMDARs (Sterratt et al., 2012). Given the results presented in this study, each of these are plausible future targets to define the detailed molecular pathway of distancedependent scaling.

\section{References}

Andrasfalvy BK, Magee JC (2001) Distance-dependent increase in AMPA receptor number in the dendrites of adult hippocampal cal pyramidal neurons. J Neurosci 21:9151-9159. Medline

Andrásfalvy BK, Smith MA, Borchardt T, Sprengel R, Magee JC (2003) Impaired regulation of synaptic strength in hippocampal neurons from glur1-deficient mice. J Physiol 552:35-45. CrossRef Medline

Andrásfalvy BK, Makara JK, Johnston D, Magee JC (2008) Altered synaptic and nonsynaptic properties of cal pyramidal neurons in kv4.2 knockout mice. J Physiol 586:3881-3892. CrossRef Medline

Bullis JB, Jones TD, Poolos NP (2007) Reversed somatodendritic i(h) gradient in a class of rat hippocampal neurons with pyramidal morphology. J Physiol 579:431-443. Medline

Gainey MA, Hurvitz-Wolff JR, Lambo ME, Turrigiano GG (2009) Synaptic scaling requires the glur2 subunit of the ampa receptor. J Neurosci 29: 6479-6489. CrossRef Medline

Gill MB, Kato AS, Roberts MF, Yu H, Wang H, Tomita S, Bredt DS (2011) Cornichon-2 modulates ampa receptor-transmembrane AMPA receptor regulatory protein assembly to dictate gating and pharmacology. J Neurosci 31:6928-6938. CrossRef Medline

Goold CP, Nicoll RA (2010) Single-cell optogenetic excitation drives homeostatic synaptic depression. Neuron 68:512-528. CrossRef Medline

Granger AJ, Shi Y, Lu W, Cerpas M, Nicoll RA (2013) Ltp requires a reserve pool of glutamate receptors independent of subunit type. Nature 493: 495-500. Medline

Hayashi Y, Shi SH, Esteban JA, Piccini A, Poncer JC, Malinow R (2000) Driving ampa receptors into synapses by LTP and CaMKII: requirement for glur 1 and PDZ domain interaction. Science 287:2262-2267. CrossRef Medline

Herring BE, Shi Y, Suh YH, Zheng CY, Blankenship SM, Roche KW, Nicoll RA (2013) Cornichon proteins determine the subunit composition of synaptic AMPA receptors. Neuron 77:1083-1096. CrossRef Medline

Katz Y, Menon V, Nicholson DA, Geinisman Y, Kath WL, Spruston N (2009) Synapse distribution suggests a two-stage model of dendritic integration in cal pyramidal neurons. Neuron 63:171-177. CrossRef Medline
Lu W, Shi Y, Jackson AC, Bjorgan K, During MJ, Sprengel R, Seeburg PH, Nicoll RA (2009) Subunit composition of synaptic ampa receptors revealed by a single-cell genetic approach. Neuron 62:254-268. CrossRef Medline

Magee JC (1999) Dendritic Ih normalizes temporal summation in hippocampal cal neurons. Nat Neurosci 2:848. CrossRef Medline

Magee JC, Cook EP (2000) Somatic EPSP amplitude is independent of synapse location in hippocampal pyramidal neurons. Nat Neurosci 3:895903. CrossRef Medline

Meng Y, Zhang Y, Jia Z (2003) Synaptic transmission and plasticity in the absence of ampa glutamate receptor glur2 and glur3. Neuron 39:163-176. CrossRef Medline

Nicholson DA, Trana R, Katz Y, Kath WL, Spruston N, Geinisman Y (2006) Distance-dependent differences in synapse number and AMPA receptor expression in hippocampal cal pyramidal neurons. Neuron 50:431-442. CrossRef Medline

Schwenk J, Harmel N, Zolles G, Bildl W, Kulik A, Heimrich B, Chisaka O, Jonas P, Schulte U, Fakler B, Klöcker N (2009) Functional proteomics identify cornichon proteins as auxiliary subunits of AMPA receptors. Science 323:1313-1319. CrossRef Medline

Shi S, Hayashi Y, Esteban JA, Malinow R (2001) Subunit-specific rules governing ampa receptor trafficking to synapses in hippocampal pyramidal neurons. Cell 105:331-343. CrossRef Medline

Shi Y, Suh YH, Milstein AD, Isozaki K, Schmid SM, Roche KW, Nicoll RA (2010) Functional comparison of the effects of TARPs and cornichons on AMPA receptor trafficking and gating. Proc Natl Acad Sci U S A 107: 16315-16319. CrossRef Medline

Smith MA, Ellis-Davies GC, Magee JC (2003) Mechanism of the distancedependent scaling of Schaffer collateral synapses in rat cal pyramidal neurons. J Physiol 548:245-258. CrossRef Medline

Sterratt DC, Groen MR, Meredith RM, van Ooyen A (2012) Spine calcium transients induced by synaptically-evoked action potentials can predict synapse location and establish synaptic democracy. PLoS Comput Biol 8:e1002545. CrossRef Medline

Turrigiano GG (2008) The self-tuning neuron: synaptic scaling of excitatory synapses. Cell 135:422-435. CrossRef Medline

Turrigiano GG, Leslie KR, Desai NS, Rutherford LC, Nelson SB (1998) Activity-dependent scaling of quantal amplitude in neocortical neurons. Nature 391:892-896. CrossRef Medline

Wayman GA, Lee YS, Tokumitsu H, Silva A, Soderling TR (2008) Calmodulin-kinases: modulators of neuronal development and plasticity. Neuron 59:914-931. CrossRef Medline

Wenthold RJ, Petralia RS, Blahos J II, and Niedzielski AS (1996) Evidence for multiple ampa receptor complexes in hippocampal ca1/ca2 neurons. J Neurosci 16:1982-1989. Medline

Williams SR, Mitchell SJ (2008) Direct measurement of somatic voltage clamp errors in central neurons. Nat Neurosci 11:790-798. CrossRef Medline

Zamanillo D, Sprengel R, Hvalby O, Jensen V, Burnashev N, Rozov A, Kaiser KM, Koster HJ, Borchardt T, Worley P, Lübke J, Frotscher M, Kelly PH, Sommer B, Andersen P, Seeburg PH, Sakmann B (1999) Importance of AMPA receptors for hippocampal synaptic plasticity but not for spatial learning. Science 284:1805-1811. CrossRef Medline 